\title{
Spatiotemporal integration of sensory stimuli in complex regional pain syndrome and dystonia
}

\author{
Monique A. van Rijn · Jacobus J. van Hilten • \\ J. Gert van Dijk
}

Received: 8 March 2009/Accepted: 20 March 2009/Published online: 16 April 2009

(c) The Author(s) 2009. This article is published with open access at Springerlink.com

\begin{abstract}
The aetiology of dystonia in complex regional pain syndrome (CRPS-I) is incompletely understood. In primary dystonia, somatosensory-evoked potentials (SSEP) after spatially or temporally separated stimulation revealed impaired central sensory integration. Information on somatosensory processing in dystonia in CRPS-I patients may provide better insight into the underlying pathophysiological mechanism. We studied SSEPs in 33 patients with CRPS-I and dystonia and 19 healthy controls. N9, N14, N20 and N35 amplitudes were recorded after paired stimulation of median and ulnar nerves ("spatial") and after stimulation of both nerves with single stimuli and with interstimulus intervals of 20 and $40 \mathrm{~ms}$ ("temporal" stimulation). Finally, both methods were integrated resulting in spatiotemporal stimulation. Statistical testing was performed using linear mixed model analysis of variance. SSEP amplitudes were significantly suppressed after spatial and temporal stimulation. No difference was observed between patients and healthy controls. Spatiotemporal stimulation did not show an additional suppressive effect in any group. Central sensory integration of proprioceptive afferent input is normal in patients with CPRS-related dystonia. Other mechanisms may underlie the development of dystonia in this disorder.
\end{abstract}

Keywords Complex regional pain syndrome . Dystonia - Somatosensory-evoked potentials .

Somatosensory system

M. A. van Rijn $(\bowtie)$ · J. J. van Hilten · J. G. van Dijk Department of Neurology and Clinical Neurophysiology, Leiden University Medical Centre, P.O. Box 9600,

2300 RC Leiden, The Netherlands

e-mail: m.a.van_rijn@lumc.nl

\section{Introduction}

Complex regional pain syndrome type 1 (CRPS) is commonly known as a disorder that is preceded by a minor to severe trauma to an extremity in the absence of an obvious nerve lesion and occurs more frequently in women (Allen et al. 1999; Schwartzman and Kerrigan 1990; Veldman et al. 1993).

There is compelling evidence indicating that aberrant inflammation plays an important role in the acute phase of CRPS (Birklein et al. 2001; Leis et al. 2004). In some patients, the acute phase of CRPS may lead to a new phase called central sensitization, which is associated with neurochemical changes, functional alterations of excitatory and inhibitory connections, cell death of neurons and interneurons and sprouting of new connections in the spinal cord (Woolf and Mannion 1999). Such changes may have important influences on sensory processing and movement control. Indeed, central sensitization is typically associated with chronic pain, allodynia and hyperalgesia, and about $20 \%$ of the CRPS patients develop dystonia that may spread to multiple extremities (van Hilten et al. 2001, 2005; Woolf and Mannion 1999). Traditionally, dystonia is associated with basal ganglia dysfunction, but recent studies have broadened the concept of dystonia by defining it as a disorder of neural circuits that mediate sensorymotor integration as opposed to a disorder of a single brain structure (Huang et al. 2006; Mink 2006; Tisch et al. 2006). In line with this new concept, several neurophysiological studies in CRPS-related dystonia have found evidence of impaired inhibition at the spinal cord and motor cortex (Schouten et al. 2003; Schwenkreis et al. 2003; van de Beek et al. 2002).

The recording of somatosensory-evoked potentials (SSEP) in a spatially or temporally separated stimuli design 
is another method to evaluate cortical disinhibition. In primary segmental or generalized dystonia, this approach has revealed evidence of impaired cortical inhibition (Frasson et al. 2001; Tinazzi et al. 2000). In one study, SSEPs were recorded after stimulating the median and ulnar nerves, both separately and simultaneously (Tinazzi et al. 2000). In normal subjects, adding SSEPs obtained separately for the two nerves resulted in amplitudes that were higher than when the two nerves were stimulated simultaneously, showing that there is a cortical "competition". In dystonia patients this effect was less pronounced, which was explained as a defect of surrounding inhibition. In another study, SSEPs were recorded after single shocks and after pairs of shocks (Frasson et al. 2001). The response to the second of the two shocks is normally lower in amplitude than that to the first one, but this effect was less pronounced in patients with dystonia, supporting the concept of cortical disinhibition. It is not known whether such changes also occur in secondary forms of dystonia such as dystonia associated with CPRS-I. The current study, therefore, applied SSEPs with temporal and spatial separated stimuli and their interactions in CRPS-I-related dystonia to evaluate the integrity of cortical proprioceptive afferent processing.

\section{Patients and methods}

Patients and controls

We studied SSEPs in 33 consecutive CRPS-I patients (Table 1, 32 women, 1 men, age range 18-60, mean age 39.7 years) in whom dystonia progressed to a multifocal or generalized distribution and 19 healthy controls (19 women, age range 23-55, mean age 40.2 years). All patients fulfilled the criteria for CPRS-I of the International Association for the Study of Pain (IASP) (Merskey and Bogduk 1994). All patients had tonic dystonia of at least two extremities, including one upper extremity. In the majority of patients, dystonia was limited to the distal extremity and mostly involved flexion of digits and wrists in the arms, and inversion and flexion postures in the feet. In a minority of patients, dystonia extended proximally to either elbows or shoulders, and knees or hips. None of the patients had a history of birth trauma or abnormal development. Other causes of dystonia had been excluded using appropriate blood and imaging studies (computed tomography, magnetic resonance imaging) of the spinal cord and brain. Patients were allowed to continue with their current medication. Informed consent was obtained according to the Declaration of Helsinki and the study was approved by the ethical committee of the Leiden University Medical Center.
Table 1 Demographics of 33 patients with CRPS and dystonia

\begin{tabular}{ll}
\hline Characteristic & Value \\
\hline Females, no. (\%) & $32(97)$ \\
Disease duration, mean (SD), years & $9.0(6.4)$ \\
Age at assessment, mean (SD), years & $39.7(10.9)$ \\
Age at onset of CRPS, mean (SD), years & $30.7(10.0)$ \\
Affected extremities with CRPS, no. & \\
2 & 10 \\
3 & 7 \\
4 & 16 \\
Affected extremities with dystonia, no. & \\
2 & 16 \\
3 & 6 \\
4 & 11 \\
Concomitant oral medication, no. $(\%)$ & \\
Antidepressants & $10(30)$ \\
Baclofen & $11(33)$ \\
Benzodiazepines & $12(36)$ \\
Anticonvulsant drug & $4(12)$ \\
Acetaminophen or NSAIDs & $14(42)$ \\
Opiods & $14(42)$ \\
\hline
\end{tabular}

SSEP acquisition

Somatosensory-evoked potentials were recorded using a Nicolet Viking III P (Nicolet Biomedical, Madison, WI, USA). Patients were instructed to lie supine on an examination couch. Electrical stimuli of $0.2 \mathrm{~ms}$ duration were given to the median and ulnar nerves at the wrist of the affected arm in the patient group and the right arm in the control group. The sampling rate was 10,000 per second. Stimulus intensity was adjusted to result in a small twitch of the hand muscles innervated by the nerve in question. Stimulation frequency was $4.7 \mathrm{~Hz}$. Each SSEP consisted of a four-channel recording $(30-1,000 \mathrm{~Hz}$ bandpass filter): Erb's point; a cervical lead aimed at the N14 peak, and the other two recorded ipsilateral and contralateral cortical activity. For all leads, a $100 \mathrm{~ms}$ period was recorded.

Somatosensory-evoked potentials were acquired with two sessions of 350 stimuli, which allowed reproducibility to be judged visually before the automated analysis (see below). We used three "temporal" settings, consisting of single shocks, paired shocks with an interstimulus interval (ISI) of $20 \mathrm{~ms}$ and paired shocks with an ISI of $40 \mathrm{~ms}$. The single shocks will further be labelled as having an ISI of $0 \mathrm{~ms}$. We used three spatial settings: stimulation of the median nerve, of the ulnar nerve and of both nerves together. All combinations were studied with nonrandom intervals, resulting in nine SSEPs. 


\section{SSEP analysis}

Responses to single stimuli were subtracted from those obtained following temporal paired stimuli (Fig. 1). This procedure resulted in SSEPs that started at the onset of the second stimulus, and that represented neural activity resulting only from the second of the paired stimuli. Afterwards, SSEPs following median and ulnar nerve
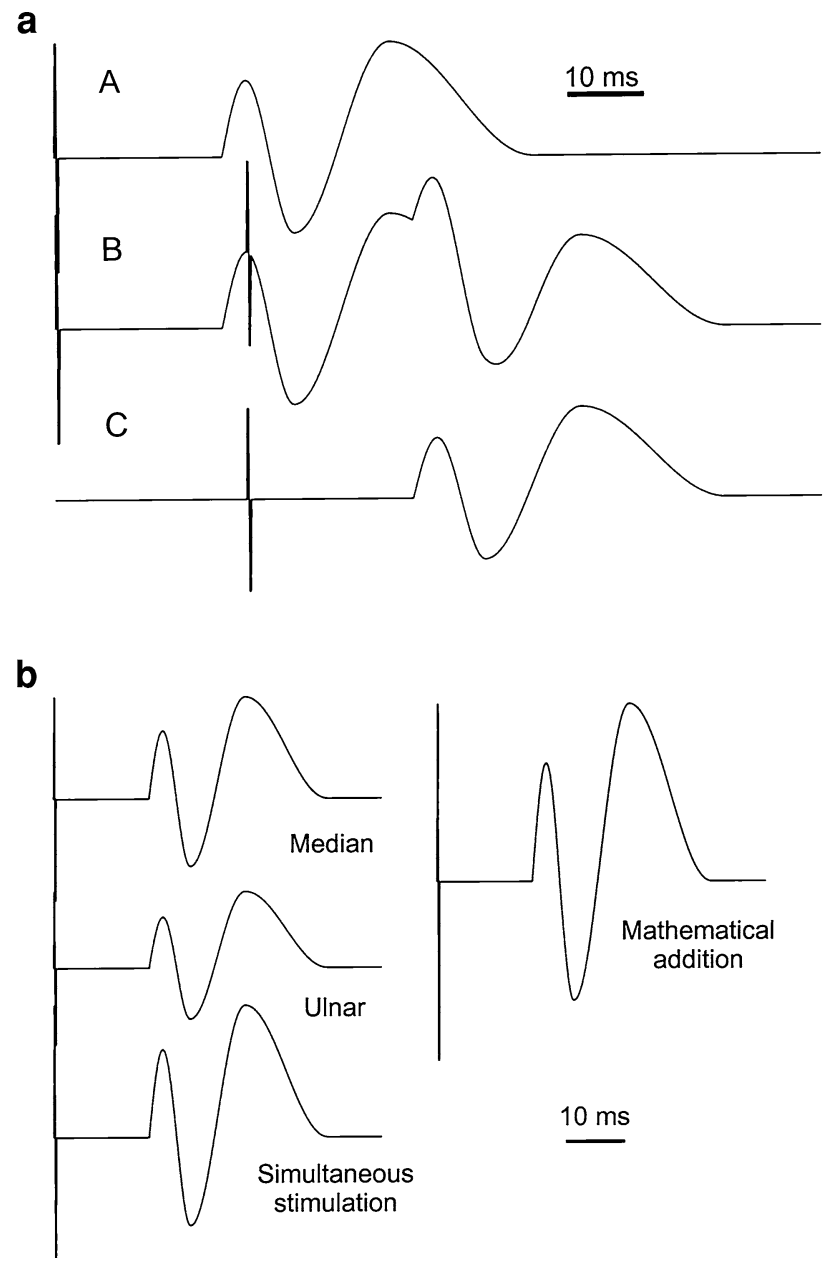

Fig. 1 Scheme of SSEP analysis. a Shows temporal effects. Simulated cortical leads are shown to explain the subtraction procedure. Panel $A$ shows an SSEP obtained after a stimulus at the beginning of the trace. Panel $B$ shows the result after paired stimulation with an interstimulus interval of $20 \mathrm{~ms}$ (recognizable through the stimulus artefact): the recording contains the added response to both stimuli. Panel $C$ shows the remainder after trace A is subtracted from trace B: the response to the first stimulus is negated, leaving an isolated response to the second stimulus. For further analysis, the first $20 \mathrm{~ms}$ were cut from the trace (not shown). b Shows spatial effects. The lefthand side of the figure shows simulated cortical SSEPs obtained for stimulation of the median nerve (top), the ulnar nerve (middle) as well as that of both nerves stimulated simultaneously (bottom). The righthand side shows the mathematical addition of the two separate and non-simultaneously acquired median and ulnar nerve SSEPs stimulation were summed separately per ISI (Fig. 2). This resulted in four groups of SSEPs representing: (1) median nerve stimulation, (2) ulnar nerve stimulation, (3) simultaneous stimulation of the median and ulnar nerves (labelled as "simultaneous") and (4) the mathematical sums of the SSEPs obtained separately for median and ulnar nerves (labelled "nonsimultaneous"). Grand averages were constructed to aid visualization of responses. The result of these procedures was 12 SSEPs per subject: for each of the three temporal conditions of ISIs $(0,20$ and $40 \mathrm{~ms}$ ), there were four spatial variants (median, ulnar, simultaneous and nonsimultaneous).

Peaks were analysed objectively using a computer programme (written in Matlab, The Mathworks, Natick, version 6.1.0.450, release 12.1). For example, Erb's peak was identified as the point of maximum electrical potential in an 8-12.5 ms window in the appropriate channel. Beginnings and end of these windows were based on inspection of grand averages. A local minimum just following the identified maximum was located in a 10$17.5 \mathrm{~ms}$ window in the same channel, and Erb's peak amplitude was defined as the difference in voltage between the two points. The N14 peak was identified using a local maximum in a $10-17.5 \mathrm{~ms}$ window, compared to a local minimum in a $12.5-22.5 \mathrm{~ms}$ window, similarly as for the Erb's peak. For the N20 peak, a 15$22.5 \mathrm{~ms}$ peak was used, and its amplitude was compared to the N25 local minimum, found with a $20-27.5 \mathrm{~ms}$ window. Additional later potentials in the cortical leads were identified using a $22.5-42.5 \mathrm{~ms}$ lead (N35) and a 32.5-50 ms window to help measure N35 amplitude. This was done for ipsilateral as well as for contralateral cortical leads. Peaks were considered absent if there was no local maximum or minimum, i.e., if the point of maximum potential coincided with an edge of the search window. Interpeak latencies were calculated for the ErbN14 latency and the N14-N20 latency.

\section{Statistical analysis}

As amplitudes and latencies showed a skewed distribution, a logarithmic transformation was performed. Subsequently, the analysis was carried out in the following order:

1. Latencies and amplitudes obtained after stimulation with single shocks of the median and ulnar nerves separately were first descriptively evaluated. Differences between SSEPs of the median and ulnar nerves were investigated with the paired samples $t$ test. Differences between patients and controls were evaluated using the $t$ test for independent samples.

2. To evaluate temporal and spatial effects of stimulation, a linear mixed model analysis of variance was 
$\mathrm{ISI}=0 ; \mathrm{M} \& \mathrm{U}$

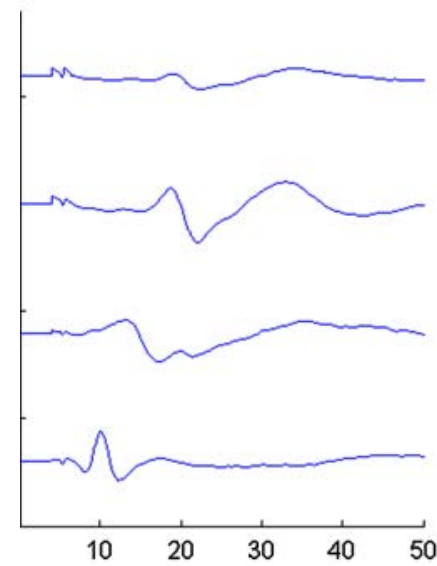

$|S|=0 ; M+U$

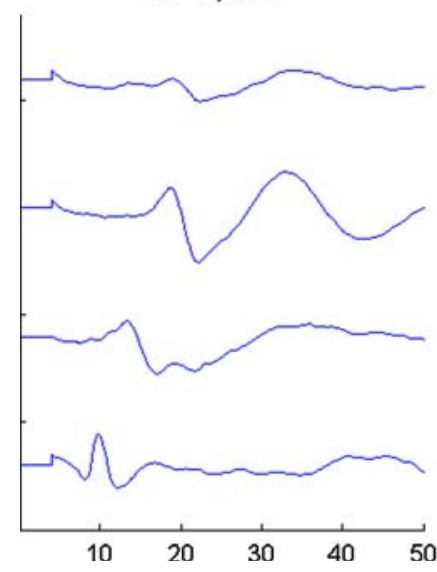

$|S|=20 ; M \& U$

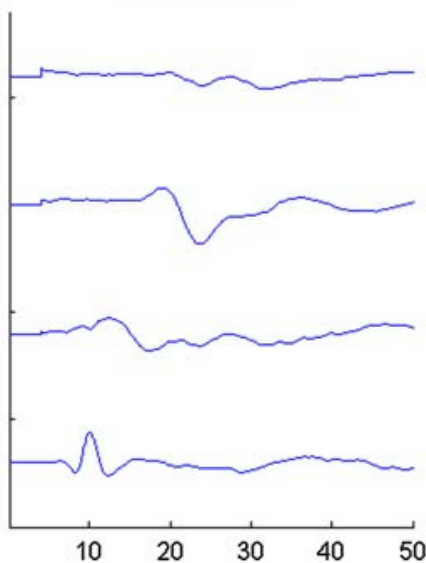

$I S I=20 ; M+U$

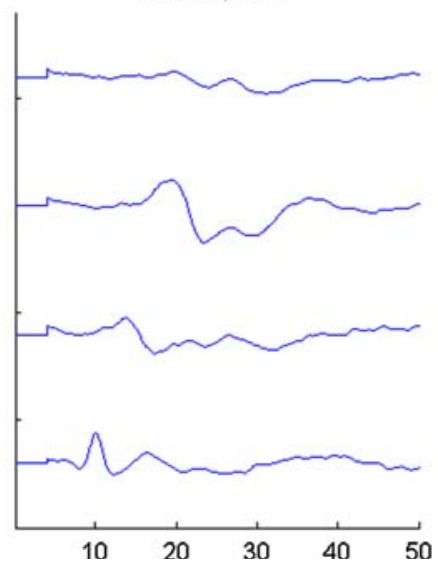

ISI=40; M\&U

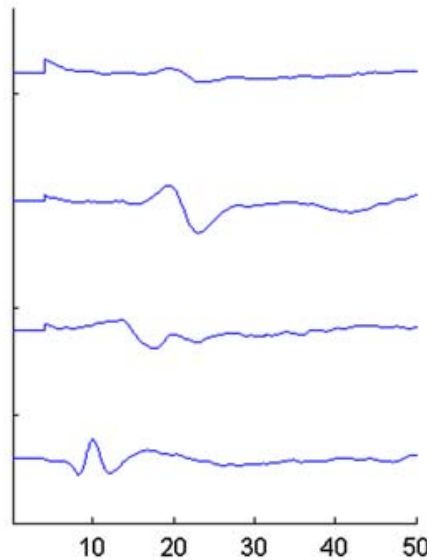

$|S|=40 ; M+U$

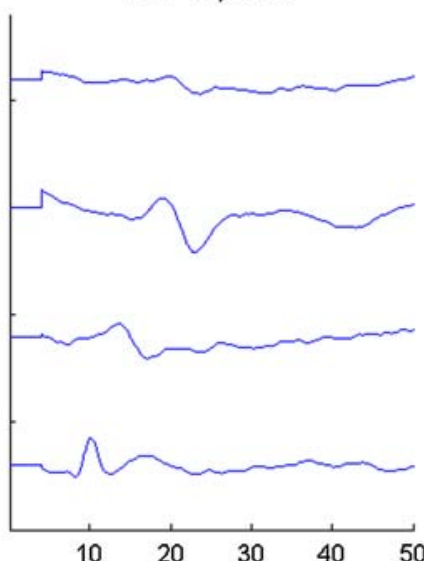

Fig. 2 Grand averages of patients' SSEPs. The six panels show grand averages for SSEPS obtained with temporal manipulation (ISI 0, 20 and $40 \mathrm{~ms}$ ) and spatial manipulation (" $M \& U$ " simultaneous stimulation of the median and ulnar nerves, " $M+U$ " mathematical sum of the SSEPs obtained with separate stimulation of the median and ulnar nerves). Horizontal scales denote ms. The four channels shown in each panel show from top to bottom, the ipsilateral cortical lead, the contralateral one, the N14 lead and the lead showing Erb's peak. Distances between ticks on the vertical axes denote $5 \mathrm{mV}$. The first $4 \mathrm{~ms}$ are set to 0 to suppress the stimulus artefact used. Amplitude of the N20 and N35 peaks were entered as the dependent variable. Spatial stimulation (simultaneous or nonsimultaneous), temporal stimulation (ISI of 0,20 or $40 \mathrm{~ms}$ ) and group (patient or control) were entered as fixed factors. The amplitude of the N9 peak was added as covariate to adjust for its possible effects on N14, N20 and N35 amplitudes.

Interactions were taken into account with all three factors (group $\times$ spatial $\times$ temporal), and when the factor group revealed a nonsignificant effect, the analysis was rerun without the three-way interaction of group, temporal stimulation and spatial stimulation. $P$ values of $<0.05$ were considered to be significant. All statistical analyses were performed with SPSS (version 12.0).

\section{Results}

Descriptive analysis

In both patients and controls, the amplitudes of the N9, N14 and N20 peaks evoked after stimulation of the median nerve were significantly higher than those evoked after stimulation of the ulnar nerve $(P<0.001$; Table 2$)$. The N35 amplitude did not differ significantly between median and ulnar nerve stimulation.

Latencies of the N14 peak were shorter after median nerve stimulation than after ulnar nerve stimulation in patients and controls (both groups; $P \leq 0.001$ ). This was also found for the N20 latency in controls $(P<0.001)$. Compared to controls, patients had a significantly shorter N9 latency after median nerve stimulation $(P=0.02)$ and 
Table 2 Median values and interquartile range of amplitudes $(\mu \mathrm{V})$ and latencies (ms) of median and ulnar nerve SEP components in controls and patients

\begin{tabular}{|c|c|c|c|c|c|c|c|c|c|}
\hline & & \multicolumn{3}{|c|}{ Control subjects } & \multicolumn{3}{|l|}{ Patients } & \multicolumn{2}{|c|}{ Patients vs controls } \\
\hline & & Median & Ulnar & $\begin{array}{l}\text { Median vs } \\
\text { ulnar } P \text { value }\end{array}$ & Median & Ulnar & $\begin{array}{l}\text { Median vs } \\
\text { ulnar } P \text { value }\end{array}$ & $\begin{array}{l}\text { Median } P \\
\text { value }\end{array}$ & $\begin{array}{l}\text { Ulnar } P \\
\text { value }\end{array}$ \\
\hline \multirow[t]{2}{*}{ N9 } & Amplitude & $3.42(2.40)$ & $1.70(1.15)$ & $<0.001$ & $2.42(2.76)$ & $1.19(1.02)$ & $<0.001$ & 0.07 & 0.38 \\
\hline & Latency & $104(5.50)$ & $108(10.0)$ & 0.12 & $101(8.8)$ & $102(9.0)$ & 0.23 & 0.04 & 0.14 \\
\hline \multirow[t]{2}{*}{ N14 } & Amplitude & $2.57(0.90)$ & $1.57(0.65)$ & $<0.001$ & $2.37(1.70)$ & $1.25(0.93)$ & 0.02 & 0.62 & 0.07 \\
\hline & Latency & $137(7.5)$ & $145(4.0)$ & $<0.001$ & $134(10.8)$ & $143(19.0)$ & 0.001 & 0.09 & 0.55 \\
\hline \multirow[t]{2}{*}{$\mathrm{N} 20$} & Amplitude & $2.59(2.55)$ & $2.42(1.29)$ & $<0.001$ & $2.92(2.53)$ & $2.28(1.87)$ & $<0.001$ & 0.76 & 0.50 \\
\hline & Latency & $191(9.0)$ & $197(5.0)$ & $<0.001$ & $189(8.8)$ & $192(14.0)$ & 0.50 & 0.39 & 0.02 \\
\hline \multirow[t]{2}{*}{ N35 } & Amplitude & $2.28(1.12)$ & $2.46(1.32)$ & 0.64 & $2.35(2.62)$ & $1.80(1.98)$ & 0.06 & 0.53 & 0.08 \\
\hline & Latency & $323(35.0)$ & $336(31.8)$ & 0.09 & $338(36.0)$ & $334(24.0)$ & 0.94 & 0.46 & 0.89 \\
\hline
\end{tabular}

a significantly shorter N20 latency after ulnar nerve stimulation $(P=0.02)$.

Spatiotemporal interaction

The temporal factor resulted in highly significant differences for all SSEP amplitudes after adjusting for the influence of the N9 amplitude $(P<0.001$ for N9, N14, N20 and N35 amplitudes). Table 3 shows that this was due to lower amplitudes following the second of the two stimuli. The spatial factor also resulted in highly significant differences for all SSEP amplitudes $(P<0.001$ for $N 9$, N14, N20 and N35 amplitudes). Simultaneous stimulation of median and ulnar nerve evoked amplitudes that were smaller than the arithmetic sum of separately obtained SSEPs of the median and ulnar nerves.

The factor group did not show a significant interaction with either the spatial or temporal factor, meaning that patients and controls responded similarly to spatial and temporal effects. The three-way interaction did not result in significant differences for any peak, so the analysis was rerun without interactions with group. The interaction between the spatial and temporal factors did not show significant differences for SSEP amplitudes $(P=0.92$, 0.27, 0.18 and 0.30 for N9, N14, N20 and N35 amplitudes, respectively). The interaction between the factor group and the factor spatiotemporal stimulation was not significant either. Within patients, there was no significant interaction between the use of benzodiazepines or baclofen and the factor of spatiotemporal stimulation.

\section{Discussion}

In patients with primary dystonia, SSEPs after spatial or temporal separated stimuli have yielded evidence of impaired cortical inhibition (Frasson et al. 2001; Tinazzi et al. 2000). Against this background, we evaluated the presence of disinhibition in the sensory cortex by studying SSEPs obtained after spatiotemporal stimuli.

Table 3 Median values and interquartile range of N20 and N35 SSEP amplitudes $(\mu \mathrm{V})$ after spatiotemporal stimulation in controls and patients

\begin{tabular}{|c|c|c|c|c|c|}
\hline & \multicolumn{5}{|c|}{ Temporal stimulation } \\
\hline & \multirow[t]{2}{*}{ SSEP } & \multirow[t]{2}{*}{ Amplitude } & \multicolumn{3}{|c|}{ Interstimulus interval } \\
\hline & & & $0 \mathrm{~ms}$ & $20 \mathrm{~ms}$ & $40 \mathrm{~ms}$ \\
\hline \multirow{4}{*}{$\begin{array}{l}\text { Simultaneous stimulation of } \\
\text { median and ulnar nerve (spatial } \\
\text { stimulation) }\end{array}$} & \multirow[t]{2}{*}{$\mathrm{N} 20$} & Controls & $3.46(3.38)$ & $3.55(4.20)$ & $2.88(3.20)$ \\
\hline & & Patients & $3.03(2.81)$ & $3.17(2.62)$ & $2.70(2.35)$ \\
\hline & \multirow[t]{2}{*}{ N35 } & Controls & $2.92(1.78)$ & $2.71(1.96)$ & 1.79 (1.49) \\
\hline & & Patients & $2.54(1.67)$ & $1.47(2.17)$ & $1.83(1.54)$ \\
\hline \multirow{4}{*}{$\begin{array}{l}\text { Mathematical sum of individual } \\
\text { stimulation of median and ulnar } \\
\text { nerve }\end{array}$} & \multirow[t]{2}{*}{$\mathrm{N} 20$} & Controls & $4.49(3.76)$ & $4.58(2.89)$ & $4.00(1.23)$ \\
\hline & & Patients & $4.33(4.05)$ & $3.51(2.92)$ & $2.82(1.66)$ \\
\hline & \multirow[t]{2}{*}{ N35 } & Controls & $4.37(2.44)$ & $3.07(2.85)$ & $2.39(2.29)$ \\
\hline & & Patients & $3.82(3.63)$ & $2.71(2.20)$ & $2.44(2.90)$ \\
\hline
\end{tabular}

The temporal factor resulted in highly significant differences for the N20 and N35 amplitudes after adjusting for the influence of the N9 amplitude $(P<0.001)$. The spatial factor also resulted in highly significant differences for the $\mathrm{N} 20$ and $\mathrm{N} 35$ amplitudes $(P<0.001)$ 
Contrary to the reported findings in patients with primary dystonia, spatial and temporal SSEP stimulation did not reveal a difference between CRPS-I patients with dystonia and controls. The temporal factor proved highly significant, in that the second of the two stimuli given with a short interval evoked a potential of lesser amplitude than the first one. As such, clear evidence of differential processing was obtained, involving habituation or inhibition of successive stimuli. Stimuli given simultaneously to two different nerves resulted in amplitudes that were smaller than the sum of two SSEPs obtained separately, indicating "competition" for cortical processing. However, both approaches did not differ between groups. As the amount of sensory input with spatiotemporal stimulation to the somatosensory system is larger than with temporal or spatial stimulation alone, one would expect additional suppression of SSEPs. However, interactions of spatial and temporal effects did not reveal an additional suppression of amplitudes, in patients or controls. Possibly, this is due to saturation or habituation of the gating capacity of the somatosensory system.

The current results thus indicate that sensory processing of proprioceptive input is normal in patients with CPRS and dystonia. One other study on CRPS patients measured EMG responses to TMS preceded by paired median nerve stimulation and found suppression similar to healthy controls, suggesting a normal sensorimotor interaction (Turton et al. 2006). Since spatial and temporal stimulation in both our groups suppressed SSEP amplitudes, methodological issues are an unlikely explanation of our findings. It is also unlikely that medication was of influence, as we found no significant effects of the use of benzodiazepines or baclofen. We do not think that that our results were influenced by ongoing dystonic contraction of the muscles in the affected arm. Gantchev et al. (1994) studied this issue in healthy subjects and found no difference between the "hold" condition (isometric contraction) and rest.

The failure to demonstrate abnormalities in our patients may be interpreted as evidence in favour of the notion that psychogenic factors contribute to the dystonia in many of these patients (Schrag et al. 2004). However, $73 \%$ of the patients in our study also participated in a case-control study, in which their psychological characteristics were compared with those of patients with affective and conversion disorders (Reedijk et al. 2008). In line with another case-control study (van der Laan et al. 1999), this study found no evidence to support a distinct psychological profile in patients with CRPS-related dystonia.

To the best of our knowledge, SSEPs after spatial or temporal separated stimuli have not been applied to other secondary causes of dystonia and it may well be that disinhibition of the sensory cortex is an exclusive finding of primary dystonia. In line with the concept of dystonia as a disorder of neural circuits that mediate sensorymotor integration, several studies have documented physiologic abnormalities at multiple levels of the central nervous system in dystonia of varying aetiology (Huang et al. 2006; Mink 2006; Tisch et al. 2006). This raises an interesting issue about the commonality of neural circuits involved in dystonia of different aetiologies. The generally disappointing responses of secondary dystonia to deep brain stimulation (Eltahawy et al. 2004) may indicate that different causes of dystonia are associated with differential circuit involvement. In CRPS-I, $\mathrm{C}$ and $\mathrm{A} \delta$ sensory nerve fibres play a role in neurogenic inflammation and are connected with spinal circuits that mediate nociceptive withdrawal reflexes (NWRs). One of the primary mediators of neurogenic inflammation, substance P (SP), may also activate SP receptors on lamina I neurons in the dorsal horn of the spinal cord and induce longterm potentiation, a form of neuronal plasticity (Schouenborg 2004). Both SP-sensitized NWRs in animal models and dystonia in CRPS-I patients respond to the $\mathrm{GABA}_{\mathrm{B}}$ agonist baclofen, which enhances spinal GABAergic inhibition (Saito et al. 1975; van Hilten et al. 2000). Hence, loss of spinal GABAergic inhibition likely is an important mechanism in this type of dystonia. As SSEPs primarily depend on conduction of proprioceptive input, we cannot exclude a role of abnormal processing of small fibre input in CPRS-related dystonia. Possibly, preferential stimulation of small fibres by means of laser-evoked potentials (Treede et al. 2003) or by stimulation with intra-epidermal needle electrodes (Inui et al. 2002) provides a better mode to establish abnormal cortical sensory processing.

Median nerve SSEP amplitudes appeared to be larger than ulnar nerve SSEP amplitudes, a finding that was reported by others (Yiannikas et al. 1983) and may be explained by that fact that the ulnar nerve, innervating fewer fingers than the median nerve, simply contains fewer sensory fibres. An alternative explanation resides in stimulus intensity: this was set on the basis of a motor response, and thresholds for sensory and motor responses might differ between the two nerves, perhaps because of different localization of sensory and motor fibres in the nerves.

In conclusion, previous and our findings may suggest that proprioceptive sensory processing in CRPS-I is unimpaired and that inhibition at a cortical level is restricted to the motor cortex. In view of the concept of dystonia as a circuit disorder, the finding of motor cortex disinhibition raises an interesting chicken and egg issue, which at this stage cannot be solved. However, in view of the peripheral initiation of the disorder, we favour a spinal origin of dystonia in CRPS-I with secondary changes at the supraspinal sites of the circuit. 
Acknowledgments This study was performed within TREND (Trauma RElated Neuronal Dysfunction), a knowledge consortium that integrates research on complex regional pain syndrome type 1 and is supported by a Dutch Government grant (BSIK03016). The authors wish to thank H. Putter from the Department of Medical Statistics of the Leiden University Medical Centre for the statistical advice.

Open Access This article is distributed under the terms of the Creative Commons Attribution Noncommercial License which permits any noncommercial use, distribution, and reproduction in any medium, provided the original author(s) and source are credited.

\section{References}

Allen G, Galer BS, Schwartz L (1999) Epidemiology of complex regional pain syndrome: a retrospective chart review of 134 patients. Pain 80:539-544

Birklein F, Schmelz M, Schifter S, Weber M (2001) The important role of neuropeptides in complex regional pain syndrome. Neurology 57:2179-2184

Eltahawy HA, Saint-Cyr J, Giladi N, Lang AE, Lozano AM (2004) Primary dystonia is more responsive than secondary dystonia to pallidal interventions: outcome after pallidotomy or pallidal deep brain stimulation. Neurosurgery 54:613-619

Frasson E, Priori A, Bertolasi L, Mauguiere F, Fiaschi A, Tinazzi M (2001) Somatosensory disinhibition in dystonia. Mov Disord 16:674-682

Gantchev G, Gavrilenko T, Concek V (1994) Somatosensory-evoked potentials modification related to isometric voluntary contraction. Int J Psychophysiol 17:191-196

Huang YZ, Trender-Gerhard I, Edwards MJ, Mir P, Rothwell JC, Bhatia KP (2006) Motor system inhibition in dopa-responsive dystonia and its modulation by treatment. Neurology 66:1088-1090

Inui K, Tran TD, Hoshiyama M, Kakigi R (2002) Preferential stimulation of A delta fibers by intra-epidermal needle electrode in humans. Pain 96:247-252

Leis S, Weber M, Schmelz M, Birklein F (2004) Facilitated neurogenic inflammation in unaffected limbs of patients with complex regional pain syndrome. Neurosci Lett 359:163-166

Merskey H, Bogduk N (1994) Complex regional pain syndromes. In: Classification of chronic pain. Descriptions of chronic pain syndromes and definitions of pain terms. IASP Press, Seattle, pp $40-43$

Mink JW (2006) Abnormal circuit function in dystonia. Neurology 66:959

Reedijk WB, van Rijn MA, Roelofs K, Tuijl JP, Marinus J, van Hilten JJ (2008) Psychological features of patients with complex regional pain syndrome type I related dystonia. Mov Disord 23:1551-1559

Saito K, Konishi S, Otsuka M (1975) Antagonism between lioresal and substance-P in rat spinal-cord. Brain Res 97:177-180

Schouenborg J (2004) Learning in sensorimotor circuits. Curr Opin Neurobiol 14:693-697
Schouten AC, van de Beek WJT, van Hilten JJ, Van der Helm FCT (2003) Proprioceptive reflexes in patients with reflex sympathetic dystrophy. Exp Brain Res 151:1-8

Schrag A, Trimble M, Quinn N, Bhatia K (2004) The syndrome of fixed dystonia: an evaluation of 103 patients. Brain 127:2360 2372

Schwartzman RJ, Kerrigan J (1990) The movement disorder of reflex sympathetic dystrophy. Neurology 40:57-61

Schwenkreis P, Janssen F, Rommel O, Pleger B, Volker B, Hosbach I, Dertwinkel R, Maier C, Tegenthoff M (2003) Bilateral motor cortex disinhibition in complex regional pain syndrome (CRPS) type I of the hand. Neurology 61:515-519

Tinazzi M, Priori A, Bertolasi L, Frasson E, Mauguiere F, Fiaschi A (2000) Abnormal central integration of a dual somatosensory input in dystonia - evidence for sensory overflow. Brain 123:4250

Tisch S, Limousin P, Rothwell JC, Asselman P, Zrinzo L, Jahanshahi M, Bhatia KP, Hariz MI (2006) Changes in forearm reciprocal inhibition following pallidal stimulation for dystonia. Neurology 66:1091-1093

Treede RD, Lorenz J, Baumgartner U (2003) Clinical usefulness of laser-evoked potentials. Clin Neurophysiol 33:303-314

Turton AJ, McCabe CS, Harris N, Filipovic SR (2006) Sensorimotor integration in complex regional pain syndrome: a transcranial magnetic stimulation study. Pain 127:270-275

van de Beek WJ, Vein A, Hilgevoord AA, van Dijk JG, van Hilten BJ (2002) Neurophysiologic aspects of patients with generalized or multifocal tonic dystonia of reflex sympathetic dystrophy. J Clin Neurophysiol 19:77-83

van der Laan L, van Spaendonck K, Horstink MW, Goris RJ (1999) The Symptom Checklist-90 Revised questionnaire: no psychological profiles in complex regional pain syndrome-dystonia. J Pain Symptom Manage 17:357-362

van Hilten BJ, van de Beek WJT, Hoff JI, Voormolen JH, Delhaas EM (2000) Intrathecal baclofen for the treatment of dystonia in patients with reflex sympathetic dystrophy. $\mathrm{N}$ Engl $\mathrm{J}$ Med 343:625-630

van Hilten JJ, van de Beek WJ, Vein AA, van Dijk JG, Middelkoop HA (2001) Clinical aspects of multifocal or generalized tonic dystonia in reflex sympathetic dystrophy. Neurology 56:17621765

van Hilten JJ, Blumberg H, Schwartzman RJ (2005) Factor IV: movement disorders and dystrophy-pathophysiology and measurement. In: Wilson PR, Stanton-Hicks M, Harden RN (eds) CRPS: current diagnosis and therapy. IASP Press, Seattle, pp $119-137$

Veldman PH, Reynen HM, Arntz IE, Goris RJ (1993) Signs and symptoms of reflex sympathetic dystrophy: prospective study of 829 patients. Lancet 342:1012-1016

Woolf CJ, Mannion RJ (1999) Neuropathic pain: aetiology, symptoms, mechanisms, and management. Lancet 353:1959-1964

Yiannikas C, Shahani BT, Young RR (1983) The investigation of traumatic lesions of the brachial plexus by electromyography and short latency somatosensory potentials evoked by stimulation of multiple nerves. J Neurol Neurosurg Psychiatry 46:10141022 